\title{
Advances in cardiac CT
}

Advances made by cardiac CT in the last decade have been unparalleled in the field of medical imaging. Breathtaking technological advances in scanner hardware such as wide-array/volume scanners, dual source, high-pitch helical mode, fast gantry rotation time, smaller detectors and improved electronics have made it easy to routinely and consistently obtain high quality images of the heart (1). There has been development of multi-energy/spectral/dual energy CT using various technologies that provides improved tissue and material characterization compared to what is possible with a conventional CT, which can be used in a variety of clinical applications including myocardial perfusion, lesion characterization, improving vascular contrast, decreasing artifacts and minimizing radiation (2). Advances in radiation dose reduction techniques such as automatic tube current modulation, ECG based tube current modulation, automatic tube current and voltage selection and several generations of iterative reconstruction algorithms have ensured that high quality cardiac images can be obtained with minimal radiation (3).

The accuracy of cardiac CT in depicting coronary artery anatomy compared to invasive coronary angiography (ICA) has now been established beyond any doubt (4), and CT outperforms all other noninvasive imaging tests. The high accuracy and negative predictive value make CT well suited to exclude obstructive coronary artery disease in the low to intermediate risk population (5). This has been applied effectively in the emergency room setting to triage patients presenting with chest pain and discharge negative patients faster, providing cost savings to the healthcare system (6). Application of CTA in the high-risk group is limited, primarily because of its lower specificity and difficulty to estimate the hemodynamic significance of intermediate stenotic lesions (7). Now there are options available to obtain functional information from CT. Myocardial perfusion CT performed using a variety of techniques has higher specificity (68-98\%) and PPV (55-94\%) in the diagnosis of myocardial ischemia than conventional CT, although there is additional radiation and contrast dose (8). CT-FFR is an advanced post-processing technique, performed either offsite or onsite, where using mathematical assumptions and modeling, computational fluid dynamic model is generated and FFR is obtained non-invasively from a routine coronary CTA. This technique has been shown to convert $68 \%$ of CT 'false positives' to true negatives, and obviating the need for ICA in $61 \%$ of individuals compared to a simple CTA based algorithm (9).

Large multicenter trials have also established the role of CT in predicting major cardiovascular events which helps in prognostication and early initiation of preventive therapy at subclinical stage. Calcium score has been convincingly shown to be useful in a wide spectrum of age and risk factors, as an adjunct to traditional risk stratification models, due to its ability to reclassify, particularly in those who in the intermediate group and are considered/recommended statins (10). A zero calcium score has been shown to give a warranty period of at least 15 years in a recent study (11). Presence of high risk plaque features such as positive remodeling and low attenuation plaques and plaque progression are important risk factors for the development of acute coronary syndrome (ACS), both at short and mid-term $(12,13)$. Even plaques without high risk factors increase the cardiovascular risk over a 5-year period based on CONFIRM registry data (14). Due to these predictive abilities, the use of CT has been shown to improve outcomes and reduce adverse events by early initiation of therapy, particularly in the recent SCOT-HEART and PROMISE trials $(15,16)$. A recent study showed that even at 80 -month follow up, the adverse event rate was lower in a treatment pathway that included cardiac CT (17).

These advances in cardiac CT and additional issues are explored in more detail in this special focused issue by several leaders in the field of cardiac CT. Dr. Achenbach's article gives a bird's eye view of the future directions of cardiac CT (18). Branch et al. discuss the technique, applications and challenges of CT myocardial perfusion in detail (19). Kueh et al. describe the principles, technique and applications of noninvasive fractional flow reserve with cardiac CT (20). Rajiah and Maroules provide a perspective on CT ischemia testing, comparing and contrasting CT perfusion, CT-fractional flow reserve and transluminal attenuation gradient (21). Kolossvary et al. provide a comprehensive review on CT based plaque imaging and risk assessment (22). Otton et al. eloquently review the technique of $3 \mathrm{~d}$ printing from cardiovascular CT and provide a practical guide on applying it in clinical practice (23). Halliburton et al. review the advances in cardiac CT reconstruction strategies (24). Scholz et al. provide practical tips on the advances in contrast injection and acquisition protocols in cardiac CT (25). Kalisz et al. illustrate the applications of cardiac CT in the evaluation of cardiomyopathies (26).

We hope that you enjoy reading this special issue and benefit from these cutting-edge topics on the state-of-the-art cardiac $\mathrm{CT}$ as much as we did. 


\section{Acknowledgements}

None.

\section{References}

1 Halliburton SS, Rajiah P. Cardiac CT scanner technology: What is new and what is next? Curr Cardiovasc Imaging Rep 2016;9:8.

2. Rajiah P, Halliburton SS. Dual energy imaging in cardiovascular CT: Current status and impact on radiation, contrast and accuracy. Curr Cardiovasc Imaging Rep 2014;7:9289.

3. Park $\mathrm{CH}$, Lee J, Oh C, et al. The feasibility of sub-millisievert coronary CT angiography with low tube voltage, prospective ECG gating, and a knowledge-based iterative model reconstruction algorithm. Int J Cardiovasc Imaging 2015;31:197-203.

4. Mowatt G, Cook JA, Hillis GS, et al. 64-slice computed tomography angiography in the diagnosis and assessment of coronary artery disease: systematic review and meta-analysis. Heart 2008;94:1386-93.

5. Gopalakrishnan P, Wilson GT, Tak T. Accuracy of multislice computed tomography coronary angiography: a pooled estimate. Cardiol Rev 2008;16:189-96.

6. Galperin-Aizenberg M, Cook TS, Hollander JE, et al. Cardiac CT angiography in the emergency department. AJR Am J Roentgenol 2015;204:463-74.

7. Meijboom WB, Van Mieghem CA, van Pelt N, et al. Comprehensive assessment of coronary artery stenoses: computed tomography coronary angiography versus conventional coronary angiography and correlation with fractional flow reserve in patients with stable angina. J Am Coll Cardiol 2008;52:636-43.

8. Varga-Szemes A, Meinerl FG, De Cecco CN, et al. CT myocardial perfusion imaging. AJR Am J Roentgenol 2015;204:487-97.

9. Douglas PS, Pontone G, Hlatky MA, et al. Clinical outcomes of fractional flow reserve by computed tomographic angiographyguided diagnostic strategies vs usual care in patients with suspected coronary artery disease: The prospective longitudinal trial of FFRCT: outcome and resource impacts study. Eur Heart J 2015;36:3359-67.

10. Nasir K, Bittencourt MS, Blaha MJ, et al. Implications of coronary artery calcium testing among statin candidates according to American College of Cardiology/American Heart Association Cholesterol Management Guidelines: MESA (Multi-Ethnic Study of Atherosclerosis). J Am Coll Cardiol 2015;66:1657-68.

11. Valenti V, Hartaigh B, Heo R, et al. A 15-year warranty period for asymptomatic individuals without coronary artery calcium: A prospective follow-up of 9,715 individuals. JACC Cardiovasc Imaging 2015;8:900-9.

12. Motoyama S, Sarai M, Harigaya H, et al. Computed tomographic angiography characteristics of atherosclerotic plaques subsequently resulting in acute coronary syndrome. J Am Coll Cardiol 2009;54:49-57.

13. Motoyama S, Ito H, Sarai M, et al. Plaque Characterization by Coronary Computed Tomography Angiography and the Likelihood of Acute Coronary Events in Mid-Term Follow-Up. J Am Coll Cardiol 2015;66:337-46.

14. Schulman-Marcus J, ó Hartaigh B, Gransar H, et al. Sex-Specific Associations Between Coronary Artery Plaque Extent and Risk of Major Adverse Cardiovascular Events: The CONFIRM Long-Term Registry. JACC Cardiovasc Imaging 2016;9:364-72.

15. SCOT-HEART investigators. CT coronary angiography in patients with suspected angina due to coronary heart disease (SCOT-HEART): an open-label, parallel-group, multicentre trial. Lancet 2015;385:2383-91.

16. Douglas PS, Hoffmann U, Patel MR, et al. Outcomes of anatomical versus functional testing for coronary artery disease. $\mathrm{N}$ Engl J Med 2015;372:1291-300.

17. Budoff MJ, Liu S, Chow D, et al. Coronary CT angiography versus standard of care strategies to evaluate patients with potential coronary artery disease; effect on long term clinical outcomes. Atherosclerosis 2014;237:494-8.

18. Achenbach S. Coronary CT angiography_future directions. Cardiovasc Diagn Ther 2017;7:432-8.

19. Branch KR, Haley RD, Bittencourt MS, et al. Myocardial computedtomography perfusion. Cardiovasc Diagn Ther 2017;7:452-62.

20. Kueh SH, Boroditsky M, Leipsic J. Fractional flow reserve computed tomography in the evaluation of coronary artery disease. Cardiovasc Diagn Ther 2017;7:463-74.

21. Rajiah P, Maroules CD. Myocardial ischemia testing with computed tomography: emerging strategies. Cardiovasc Diagn Ther 2017;7:475-88. 
22. Kolossváry M, Szilveszter B, Merkely B, et al. Plaque imaging with CT - a comprehensive review on coronary CT angiography based risk assessment. Cardiovasc Diagn Ther 2017;7:489-506.

23. Otton JM, Birbara NS, Hussain T, et al. 3D printing from cardiovascular CT: a practical guide and review. Cardiovasc Diagn Ther 2017;7:507-26.

24. Halliburton SS, Tanabe Y, Partovi S, et al. The role of advanced reconstruction algorithms in cardiac CT. Cardiovasc Diagn Ther 2017;7:527-38.

25. Scholtz JE, Ghoshhajra B. Advances in cardiac CT contrast injection and acquisition protocols. Cardiovasc Diagn Ther 2017;7:439-51.

26. Kalisz K, Rajiah P. Computed tomography of cardiomyopathies. Cardiovasc Diagn Ther 2017;7:539-56.

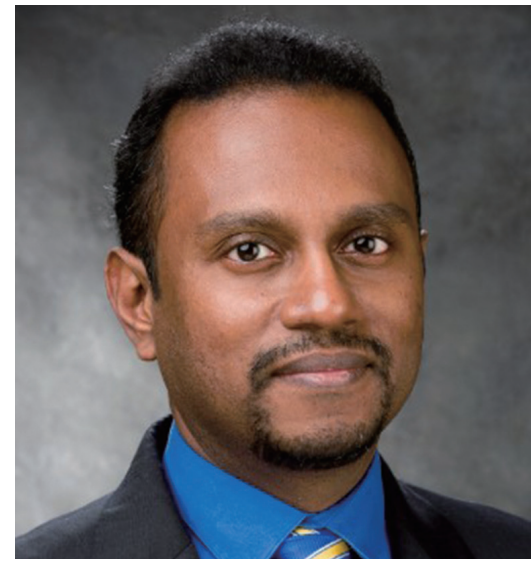

Prabhakar Rajiah, MBBS, MD, FRCR

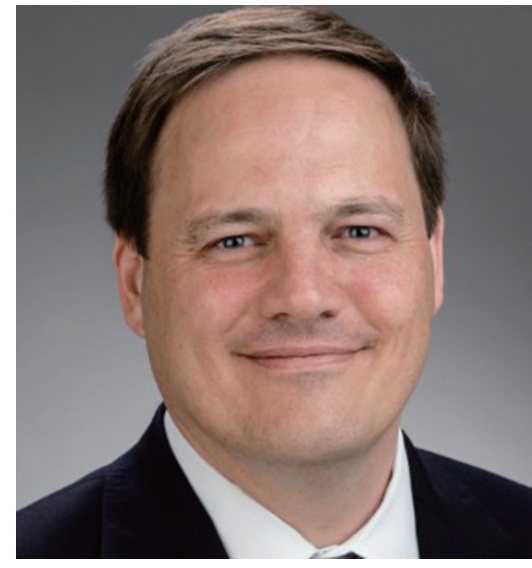

Suhny Abbara, MD

Prabhakar Rajiah, MBBS, MD, FRCR

Associate Professor of Radiology, Associate Director, Cardiac CT and MRI, UT Southwestern Medical Center, Dallas, Texas 75390, USA. (Email: radprabhakar@gmail.com)

Suhny Abbara, MD

Professor, Department of Radiology, Chief Cardiothoracic Imaging, UT Soutbwestern Medical Center, Dallas, Texas 75390, USA. (Email: subny.Abbara@utsoutbwestern.edu) doi: $10.21037 / \mathrm{cdt} .2017 .08 .11$

Conflicts of Interest: The authors have no conflicts of interest to declare.

View this article at: http://dx.doi.org/10.21037/cdt.2017.08.11

Cite this article as: Rajiah P, Abbara S. Advances in cardiac CT. Cardiovasc Diagn Ther 2017;7(5):429-431. doi: 10.21037/ cdt.2017.08.11 